\title{
3D Face Recognition Using Anthropometric and Curvelet Features Fusion
}

\author{
Dan Song, ${ }^{1,2}$ Jing Luo, ${ }^{1,2,3}$ Chunyuan $\mathrm{Zi}^{1,2}$ and Huixin Tian ${ }^{1,2}$ \\ ${ }^{1}$ College of Electrical Engineering and Automation, Tianjin Polytechnic University, Tianjin 300387, China \\ ${ }^{2}$ Key Laboratory of Advanced Electrical Engineering and Energy Technology, Tianjin 300387, China \\ ${ }^{3}$ School of Electrical, Computer and Telecommunications Engineering, University of Wollongong, Sydney, NSW 2522, Australia \\ Correspondence should be addressed to Dan Song; sweet0502@sina.com
}

Received 30 June 2015; Revised 22 September 2015; Accepted 7 October 2015

Academic Editor: Pietro Siciliano

Copyright (C) 2016 Dan Song et al. This is an open access article distributed under the Creative Commons Attribution License, which permits unrestricted use, distribution, and reproduction in any medium, provided the original work is properly cited.

Curvelet transform can describe the signal by multiple scales, and multiple directions. In order to improve the performance of 3D face recognition algorithm, we proposed an Anthropometric and Curvelet features fusion-based algorithm for 3D face recognition (Anthropometric Curvelet Fusion Face Recognition, ACFFR). First, the eyes, nose, and mouth feature regions are extracted by the Anthropometric characteristics and curvature features of the human face. Second, Curvelet energy features of the facial feature regions at different scales and different directions are extracted by Curvelet transform. At last, Euclidean distance is used as the similarity between template and objectives. To verify the performance, the proposed algorithm is compared with Anthroface3D and Curveletface3D on the Texas 3D FR database. The experimental results have shown that the proposed algorithm performs well, with equal error rate of $1.75 \%$ and accuracy of $97.0 \%$. The algorithm we proposed in this paper has better robustness to expression and light changes than Anthroface3D and Curveletface3D.

\section{Introduction}

Face recognition is widely used in video, passports, security, psychological research, automatic aid, robotics, fatigue testing, human-machine interfaces, and other occasions. In the past few decades, researchers focused on the study of $2 \mathrm{D}$ face recognition. However, 2D face recognition still has limitation to pose, expression, illumination, age variation, and other external factors. Researchers are now paying more attention to $3 \mathrm{D}$ face recognition which can overcome the pose and illumination existing in 2D face recognition. Besides, the 3D data contains more geometric information for face recognition such as geodesic distance and curvature characteristics. So, the scholars have carried on the research of $3 \mathrm{D}$ face recognition and achieved some results in recent years [1-11].

Currently, there are many researches on 3D face recognition algorithm which can be mainly divided into two categories: global feature and local feature method. Global feature method uses a unified facial expression model to represent human face, such as eigenface [5], Fisher face [6], and ICP techniques [7]. In local features method, the features of partial face region are used for face recognition, such as Gabor feature [8], Iso geodesic stripes characteristics [1], texture + Curvelet features $[9,10]$, and Anthropometric characteristics [11]. Compared with global feature method, local feature has greater advantages in dealing with expressions, gestures, light and scale change, and other issues.

Gupta et al. [11] proposed Anthropometric 3D face recognition algorithm (Anthropometric 3D Face Recognition, Anthroface3D). According to Anthropometric principle, 10 facial basis points were orientated accurately and the $3 \mathrm{D}$ geodesic distances between these points were used for human face recognition. The Anthroface3D recognition algorithm performs well (equal error rate of $1.98 \%$ and a rank 1 recognition rate of $96.8 \%$ ) in Texas 3D database.

Elaiwat et al. [9] proposed a face recognition algorithm fusing the information of $2 \mathrm{D}$ texture and $3 \mathrm{D}$ Curvelet in Curvelet domain. First, the rigid region which corresponds 
to face region except for the mouth and the area around it of $2 \mathrm{D}$ image and $3 \mathrm{D}$ image is decomposed at multiple scales and multiple directions. Second, Curvelet coefficients of the rigid region are obtained by PCA, and the key points are located by fusing the 2D Curvelet coefficients and $3 \mathrm{D}$ Curvelet coefficients. Then, the local descriptor of the key points is obtained in Curvelet domain, and recognition is achieved by Mahalanobis for 4 regions which are 3D scale 2, $3 \mathrm{D}$ scale $3,2 \mathrm{D}$ scale 2 , and 2D scale 3 , respectively. Last, 3D face recognition is realized by fusing the results of 4 regions in decision level.

Inspired by Elaiwat et al. [9] and Gupta et al. [11], this paper proposed a 3D face recognition algorithm using Anthropometric and Curvelet fusion (Anthropometric Curvelet Fusion Face Recognition, ACFFR). Due to the good representation of Anthropometric characteristics and Curvelet feature on the surface information, we extract the left eye, right eye, nose, and mouth region from the face as the local feature region for the $3 \mathrm{D}$ face recognition according to the Anthropometric characteristics. Then, the Curvelet feature vector is constructed which can describe the 4 regions at different scales and different orientations. Finally, the 3D face recognition is realized by the Euclidean distance. The Anthropometric characteristic was used to extract the nose, mouth, left eye, and right eye feature region in the proposed ACFFR algorithm. And it has better robustness for the changes of expression and illumination compared with the method proposed by Elaiwat et al. [9]. In order to verify the performance, the proposed ACFFR algorithm is tested in the famous Texas 3D database [12] and compared with the Anthroface3D and Curveletface3D face recognition methods. Experimental results show that the proposed ACFFR algorithm has better robustness in expression, light, and other environmental changes.

\section{Location of Facial Feature Region}

The selection of facial feature region is not arbitrary, which satisfies the usefulness, robustness, significance, and so on. The nose, eyes, and mouth are salient. So, the nose, eyes, and mouth region are selected as the facial feature region in our proposed method.

Figure 1 is depth image corresponding to the 15 th $3 \mathrm{D}$ face image in Texas 3D FR database [12]. It covers the major curve information of the face surface and can reflect the structural information of the whole face. The location of nose region begins with the detection of the tip of the nose, and the tip location is then employed to detect the nose width. Nose region is determined by these 3 points. These 3 points are then employed to detect the inner corners of the eyes; then, the eyes region can be determined. The location method of 4 facial feature regions is as follows.

\subsection{Location of Nose Region}

(a) Location of Nose Tip (prn). Set $A(x, y,(x, y))$ represents a point on the depth image; the location of nose tip can be realized by Gauss curvature $(K)$ and average curvature $(H)$

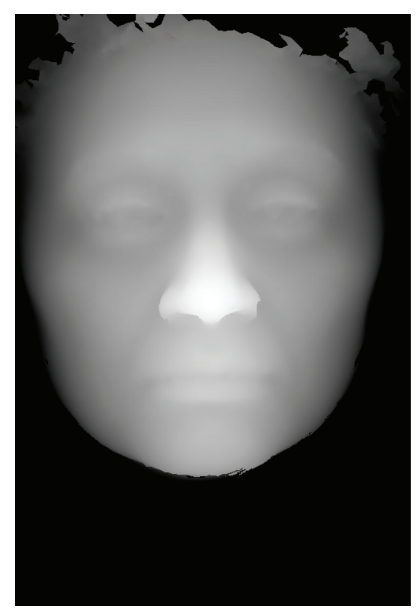

FIgURE 1: 3D facial depth image.

of face surface. $K$ and $H$ can be calculated as shown in the following formulae:

$$
\begin{aligned}
& K=\frac{z_{x x} z_{y y}-z_{x y}^{2}}{\left(1+z_{x}^{2}+z_{y}^{2}\right)^{2}} \\
& H=\frac{z_{x x}\left(1+z_{y}^{2}\right)+z_{y y}\left(1+x_{x}^{2}\right)-2 z_{x} z_{y} z_{x y}}{\left(1+z_{x}^{2}+z_{y}^{2}\right)^{3 / 2}},
\end{aligned}
$$

where $z_{x}$ and $z_{y}$ are the first partial derivatives of $z(x, y)$, with regard to $x$ and $y$, respectively, and $z_{x x}, z_{y y}$, and $z_{x y}$ are the second partial derivatives of $z(x, y)$ with regard to $x$ and $y$.

When $K>0$ and $H<0$, absolute values of $K$ and $H$ are maximum, corresponding to the location of the nose tip ( $p r n$ ) [11].

(b) Location of Nose Width Points (al-al). On average, human has nose width $\mu=35 \mathrm{~mm}$ with $\sigma=2.5 \mathrm{~mm}$ and height $\mu=$ $53 \mathrm{~mm}$ with $\sigma=3.4 \mathrm{~mm}$ [13]. The location of the nose width points in Texas 3D FR database is as follows [11]. Hence, to account for variations in the human, we fixed the width of the search region for points ( $a l-a l$ ) about the tip of the nose at $\mu+6 \sigma$. Similarly, we fixed the height of the search region at $0.6 \times(\mu+6 \sigma)$. Second, within the search region, the ACFFR $3 \mathrm{D}$ algorithm detects edges on the facial range images using Laplacian of Gaussian edge detector. Figure 2 is the edges of the search region. At last, the leftmost and rightmost points are closest to the tip of the nose along the vertical direction. The points we get are the nose width (al-al).

(c) Location of Nose Region. After steps (a) and (b), the location of the nose tip and the nose width points is acquired. The nose region is acquired according to the three points (prn, al-al) and the nose height. Then, the nose region is normalized to $100 \times \mathrm{NaN}$. Figure 3 is the location result of nose region.

However, $\mathrm{NaN}$ is obtained as a result of being mathematically undefined. 


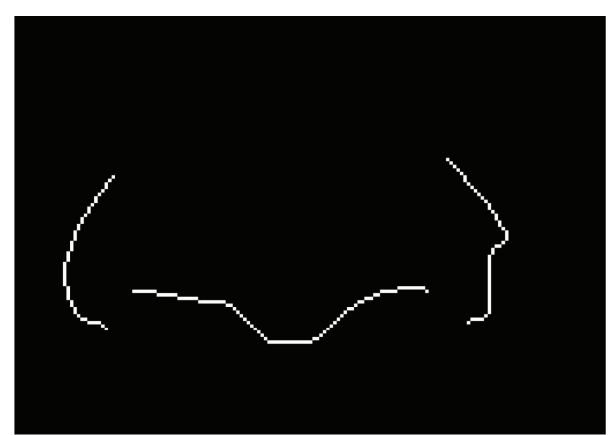

Figure 2: Edge detection result of nose region.

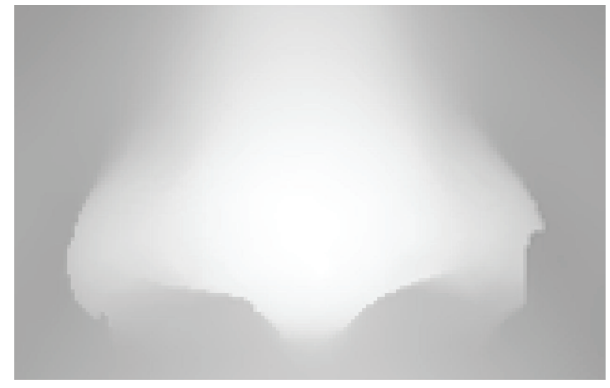

FIGURE 3: Location result of nose region.

\subsection{The Location of Left and Right Eye Region}

(a) Location of Inner Eye Corners (en-en). For an adult, the vertical distance between the inner corners of the eyes and the tip of the nose is 0.3803 times the vertical distance between the top of the head and the tip of the nose [14].

Firstly, the upper limit of the search region is set as formula (3) [11] and the lower limit as formula (4) [11]. Hence,

$$
\begin{gathered}
\operatorname{prn}_{y}+0.3803 \times 1.5 \times\left|p r n_{y}-v_{y}\right|, \\
p r n_{y}+0.3803 \times 0.33 \times\left|p r n_{y}-v_{y}\right|,
\end{gathered}
$$

where $p r n_{y}$ is the vertical coordinate of the nose tip and $v_{y}$ is the vertical coordinate of the highest point of the 3D model.

Secondly, according to the ratio of the horizontal distance between the inner corners of the eyes to nose width [13], the horizontal limit of the two search regions is determined. The limit of the inner corner of the left eye is as formula (5) [11], and that of the right eye is as formula (6) [11]. Hence,

$$
\begin{aligned}
& a l_{x, \text { left }}+0.5 \times\left|a l_{x, \text { left }}-a l_{x, \text { right }}\right|, \\
& a l_{x, \text { right }}-0.5 \times\left|a l_{x, \text { left }}-a l_{x, \text { right }}\right|,
\end{aligned}
$$

where $a l_{x, \text { left }}$ is the horizontal coordinate of the left of the nose width points and $a l_{x, \text { right }}$ is the horizontal coordinate of the right of the nose width points.

Finally, in the search region, when $K<0, K$ are the maximum absolute value, corresponding to the location of inner corners of the eyes (en-en) [11].

(b) Location of Outer Eye Corners (ex-ex). For an adult, the distance between the inner and the outer corner of an eye is approximately equal to the distance between the inner corners of the two eyes [13]. The position of the outer corner of the left eye is approximate (ex) as formula (7) [11], and the position of the outer corner of the right eye $(e x)$ is approximate as formula (8) [11]. Hence,

$$
\begin{aligned}
& \left(e n_{x, \text { left }}+\left|e n_{x, \text { left }}-e n_{x, \text { right }}\right|, \frac{\left(e n_{y, \text { left }}+e n_{y, \text { right }}\right)}{2}\right), \\
& \left(e n_{x, \text { right }}-\left|e n_{x, \text { left }}-e n_{x, \text { right }}\right|, \frac{\left(e n_{y, \text { left }}+e n_{y, \text { right }}\right)}{2}\right),
\end{aligned}
$$

where $e n_{x, \text { left }}$ is the horizontal coordinate of the left inner eye corner, $e n_{x, \text { right }}$ is the horizontal coordinate of the right inner eye corner, $e n_{y \text {,left }}$ is the vertical coordinate of the left inner eye corner, and $e n_{y, \text { right }}$ is the vertical coordinate of the right inner eye corner. The locations of the inner eye corners and the outer eye corners in Texas 3D FR database are above [11].

(c) Location of Left and Right Eye Region. After (a) and (b), the location of the inner eye corners and the outer eye corners is acquired. The location of eye regions is acquired according to the four points (en-en, ex-ex). Then, the region is normalized to $100 \times \mathrm{NaN}$. Figures 4 and 5 are the location result of left and right region.

However, $\mathrm{NaN}$ is obtained as a result of being mathematically undefined.

2.3. Location of Mouth Region. Studying the curvature of facial surface regions located below the nose, we found that the outer corners of the mouth were distinct concavities. So, we can get the location of mouth corner by calculating $H$ [11]. And the location of the detected points $(a l-a l)$ is employed to horizontally constrain these search regions.

Firstly, the search region of mouth is determined. The limitation of left mouth corner is as formula (9) [11], and the limitation of right mouth corner is as formula (10) [11]. Hence,

$$
\begin{aligned}
& a l_{x, \text { left }}+0.7 \times\left|a l_{x, \text { left }}-a l_{x, \text { right }}\right|, \\
& a l_{x, \text { right }}-0.7 \times\left|a l_{x, \text { left }}-a l_{x, \text { right }}\right|,
\end{aligned}
$$

where $a l_{x \text {,left }}$ is the horizontal coordinate of the left of the nose points and $a l_{x, \text { right }}$ is the horizontal coordinate of the right of the nose points.

Secondly, the upper and lower edge are determined. In the search region, when $H>0$, and $H$ is the maximum absolute value, the region corresponds to the location of mouth corners (ch-ch) [11].

Finally, the location of the mouth corners is acquired; the mouth region is acquired according to the mouth corners (chch) and the upper and lower edge of mouth. Then, the mouth region is normalized to $100 \times \mathrm{NaN}$. Figure 6 is the location result of mouth region.

However, $\mathrm{NaN}$ is obtained as a result of being mathematically undefined. 


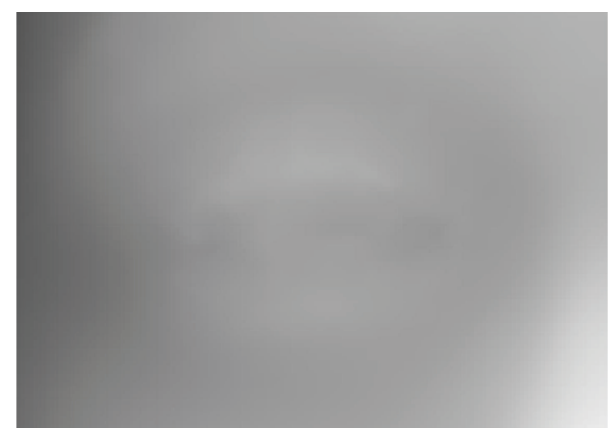

FIGURE 4: Location result of left eye region.

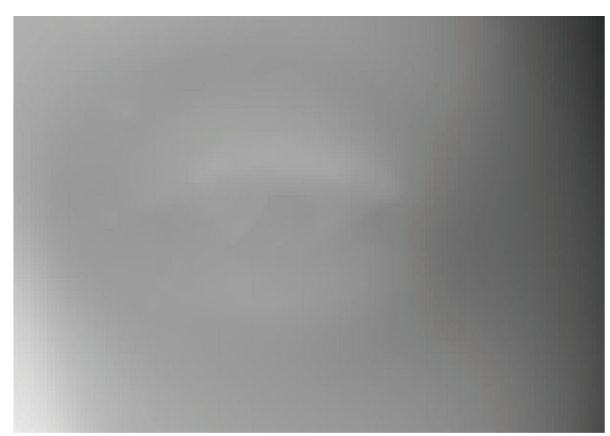

FIGURE 5: Location result of right eye region.

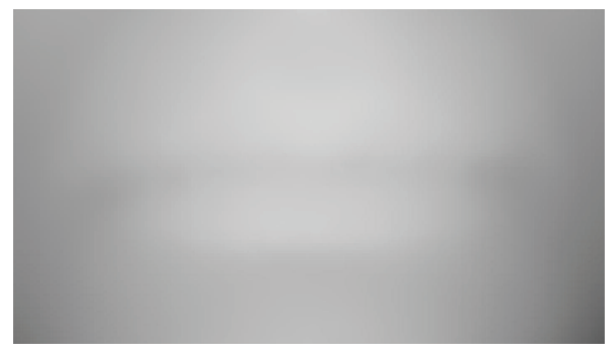

FIGURE 6: Location result of mouth region.

\section{Feature Extraction}

The Curvelet transform is a kind of multiscale transformation, which is proposed by the famous scholars Donoho and Duncan in 2000 [15]. In essence, Curvelet transform is multiscale pyramid decomposition, and each of which corresponds to the image at different directions and scales. However, this pyramid is nonstandard, the length and width of each Curvelet are variable, and the width is the square of the length. With the increase of the decomposition level, the direction of the decomposition of the Curvelet is much finer.

3.1. Acquisition of Curvelet Coefficients. After the successful localization of the facial features, the 4 normalized feature regions are decomposed by FDCT WARPING. The decomposition level is 4 .
The process of Curvelet decomposition is as follows.

Step 1. 2DFFT (fast Fourier transform) is applied on $f\left(t_{1}, t_{2}\right)$ to obtain Fourier samples $\widehat{f}\left[n_{1}, n_{2}\right]$.

Step 2. Acquire the Curvelet coefficients at scale 4, denoted by matrix $C_{4,1}$ :

(1) The right and left windows along the horizontal direction are constructed, denoted by row vector $W_{r-1}$ and $W_{l-1}$, respectively.

(2) The right and left windows along the vertical direction are constructed, denoted by row vector $W_{r-2}$ and $W_{l-2}$, respectively.

(3) The two sub-low-pass filters are constructed, denoted by row vector $f_{\text {lowpasssub1 }}$ and $f_{\text {lowpasssub2 }}$, respectively.

(4) A low-pass filter at scale 4 is constructed, denoted by matrix $\left(f_{\text {lowpass_ } 4}\right)_{\left[2 \times \text { floor }\left(2 \times M_{1}\right)+1\right] \times\left[2 \times \text { floor }\left(2 \times M_{2}\right)+1\right]}$.

(5) A two-high-pass filter $f_{\text {hipass_4 }}$ is constructed.

(6) $\hat{f}\left[n_{1}, n_{2}\right]$ is filtered by $f_{\text {hipass_4 }}$, hence generating the filtered high-pass signal at scale $4 f_{\text {hipass_4 }}^{\prime}$.

(7) Inverse 2DFFT (inverse fast Fourier transform) is applied to $f_{\text {hipass_4 }}^{\prime}$, hence generating the discrete Curvelet coefficients at scale $4, C_{4,1}$.

(8) $\hat{f}\left[n_{1}, n_{2}\right]$ is filtered by $f_{\text {hipass_4 }}$, hence generating the filtered low-pass signal $f_{\text {hipass_4 }}^{\prime}$ at scale 4 .

Step 3. The Curvelet coefficients at scale 3 and angle 1 to angle 32 are acquired.

Firstly, Curvelet coefficients at scale 3 and angle $1, C_{3,1}$, are acquired:

(1) A low-pass filter at scale 3 and angle 1 in the same way as at scale $4,\left(f_{\text {lowpass_3 } 3}\right)_{\left[2 \times \text { floor }\left(2 \times M_{1}\right)+1\right] \times\left[2 \times \text { floor }\left(2 \times M_{2}\right)+1\right]}$, is constructed.

(2) A high-pass filter at scale 3 and angle 1 in the same way as at scale 4, $\left(f_{\text {highpass_3 }}\right)_{\left[2 \times \text { floor }\left(2 \times M_{1}\right)+1\right] \times\left[2 \times \text { floor }\left(2 \times M_{2}\right)+1\right]}$, is constructed.

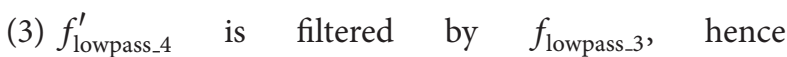
generating the filtered low-pass signal at scale 3 , $\left(f_{\text {lowpass_3 }}^{\prime}\right)_{\left[2 \times \text { floor }\left(2 \times M_{1}\right)+1\right] \times\left[2 \times \text { floor }\left(2 \times M_{2}\right)+1\right]}$.

(4) $f_{\text {lowpass_4 }}^{\prime}$ is filtered by $f_{\text {hipass_3 }}$, hence generating the filtered high-pass signal at scale 3 , which has the same size as that of $f_{\text {lowpass_4 }}^{\prime}$.

(5) The discrete locating window of wedge wave at scale 3 and angle 1 is determined. The Curvelet coefficients at scale 3 are divided into the 4 quadrants. Each quadrant has 8 angles.

(6) The discrete locating window of wedge wave $W_{\text {data }}$ is filtered and rotated, hence generating matrix $W_{\text {data_2 }}$.

(7) Inverse $2 \mathrm{DFFT}$ is applied to $W_{\text {data } 2 \text {, hence generating }}$ the Curvelet coefficient at scale 3 and angle $1, C_{3,1}$. 
TABLE 1: The Curvelet coefficient of nose region.

\begin{tabular}{|c|c|c|}
\hline Scale & Direction & Size \\
\hline 1 & 1 & $17 \times 27$ \\
\hline 2 & 16 & $\begin{array}{l}14 \times 28,12 \times 26,12 \times 26,14 \times 28 \\
17 \times 22,17 \times 20,17 \times 20,17 \times 22 \\
14 \times 28,12 \times 26,12 \times 26,14 \times 28 \\
17 \times 22,17 \times 20,17 \times 20,17 \times 22\end{array}$ \\
\hline 3 & 32 & $\begin{array}{c}27 \times 28,25 \times 27,25 \times 27,25 \times 26,25 \times 26,25 \times 27,25 \times 27,27 \times 28 \\
17 \times 41,18 \times 39,18 \times 39,17 \times 39,17 \times 39,18 \times 39,18 \times 39,17 \times 41 \\
27 \times 28,25 \times 27,25 \times 27,25 \times 26,25 \times 26,25 \times 27,25 \times 27,27 \times 28 \\
17 \times 41,18 \times 39,18 \times 39,17 \times 39,17 \times 39,18 \times 39,18 \times 39,17 \times 41\end{array}$ \\
\hline 4 & 1 & $100 \times 158$ \\
\hline
\end{tabular}
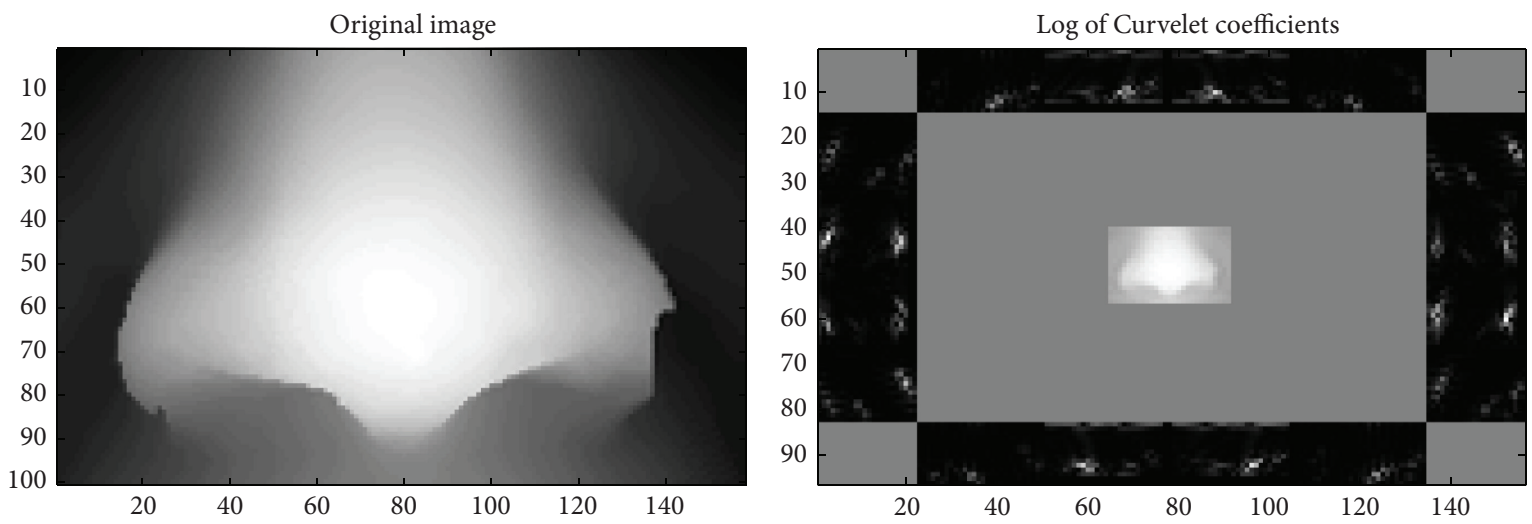

FIGURE 7: Nose region and the corresponding Curvelet decomposition.

(8) (5), (6), and (7) in Step 3 are repeated, in the same way of acquiring $C_{3,1}$; Curvelet coefficients at scale 3 and angle from 2 to 8 are generated.

(9) The Curvelet coefficients at scale 3 and the other three quadrants are acquired in the same way as that in the first quadrants.

Step 4. Step 3 is repeated, hence generating the Curvelet coefficients at scale 2 and angle from 1 to 16.

Step 5. Inverse 2DFFT is applied to the low-pass signal at scale $2, f_{\text {lowpass_2 }}^{\prime}\left(n_{1}, n_{2}\right)$, generating the Curvelet coefficient at scale $1, C_{1,1}$.

Take the nose region as an example; the size of all the coefficients at different scales and directions is shown in Table 1, and the curves at different scales and directions are shown in Figure 7.

In Figure 7, the white part of the image described the edges of nose at different directions. Meanwhile, they are important Curvelet coefficient regions of the image. Low frequency coefficient (coarse scale coefficient, the third scale coefficient) located the center of the Curvelet coefficient image. The outside scale corresponds to the high frequency coefficient (fine scale coefficient, the second scale coefficient). The second scale includes 4 strips, corresponding to scale 2
Curvelet coefficient of the four quadrants, respectively. Each subsegmented block corresponds to the corresponding scale and direction.

3.2. Extraction of Curvelet Feature. During the Curvelet decomposition, the number of directions at the fourth scale is 1. Meanwhile, the number of directions at the second scale is 16. The Curvelet coefficients at different scales and different directions represent the image direction. Thus, the direction and detail of the original signal can be approximated by Curvelet decomposition coefficients of each subblock. According to this principle, the Curvelet coefficients of each subblock are extracted by average $l_{1}$ norm in our proposed method. The formula is as follows:

$$
E_{K}=\frac{1}{M N} \sum_{i}^{M} \sum_{j}^{N}\left|x_{k}(i, j)\right|,
$$

where $\left|x_{k}(i, j)\right|$ is the modular for $x_{k}$.

The feature vector of each subblock includes 50 features, which is not only the expression of the whole instruction information for human face but also the accurate expression for the information of defective detail and direction. The feature vector of each subblock can be represented as $f=$ $\left[f_{1}, f_{2}, f_{3}, \ldots, f_{49}, f_{50}\right]$. For example, the Curvelet energy at each scale and each direction in the nose region is shown in Table 2. 
TABLE 2: The Curvelet feature of nose region.

\begin{tabular}{|c|c|}
\hline Feature & Value \\
\hline 1 & $2.5804 e+04$ \\
\hline 2 & 34.1803 \\
\hline 3 & 50.9187 \\
\hline 4 & 53.7083 \\
\hline 5 & 26.1953 \\
\hline 6 & 40.8931 \\
\hline 7 & 47.3235 \\
\hline 8 & 65.6406 \\
\hline 9 & 30.5568 \\
\hline 10 & 36.1360 \\
\hline 11 & 55.2473 \\
\hline 12 & 59.4573 \\
\hline 13 & 28.4920 \\
\hline 14 & 41.9344 \\
\hline 15 & 52.5322 \\
\hline 16 & 74.1031 \\
\hline 17 & 30.9100 \\
\hline 18 & 11.8341 \\
\hline 19 & 21.5747 \\
\hline 20 & 23.5416 \\
\hline 21 & 37.7782 \\
\hline 22 & 38.7422 \\
\hline 23 & 16.1082 \\
\hline 24 & 12.3745 \\
\hline 25 & 9.8717 \\
\hline 26 & 9.3143 \\
\hline 27 & 19.7402 \\
\hline 28 & 14.7898 \\
\hline 29 & 26.1574 \\
\hline 30 & 32.6017 \\
\hline 31 & 26.3473 \\
\hline 32 & 13.0029 \\
\hline 33 & 8.7559 \\
\hline 34 & 11.8296 \\
\hline 35 & 20.1389 \\
\hline 36 & 23.9574 \\
\hline 37 & 34.5658 \\
\hline 38 & 35.8749 \\
\hline 39 & 15.2910 \\
\hline 40 & 10.5638 \\
\hline 41 & 9.5850 \\
\hline 42 & 9.2941 \\
\hline 43 & 21.4263 \\
\hline 44 & 17.5710 \\
\hline 45 & 27.3523 \\
\hline 46 & 33.7251 \\
\hline 47 & 24.1446 \\
\hline 48 & 12.4393 \\
\hline 49 & 8.6808 \\
\hline 50 & 71.7929 \\
\hline
\end{tabular}

Considering that there are 50 features in each region, a total of 200 features are extracted for $3 \mathrm{D}$ face recognition. Finally, the template matching method is used to accomplish the recognition.
TABLE 3: The training set and test set.

\begin{tabular}{lcccc}
\hline & \multirow{2}{*}{ Body number } & \multicolumn{3}{c}{ The total number of face images } \\
& & Neutral & Expression & All \\
\hline $\begin{array}{l}\text { Training set } \\
\text { Test set }\end{array}$ & 20 & 250 & 200 & 450 \\
$\quad$ Model set & 103 & 103 & 0 & 103 \\
$\quad$ Target subset & 95 & 395 & 205 & 600 \\
\hline
\end{tabular}

3.3. Template Matching. By the method mentioned above, we can get 4 feature regions of each image and 50 features in every feature region. The Euclidean distance between the target and the template is used to accomplish the $3 \mathrm{D}$ face recognition. The calculation of Euclidean distance $d$ is shown in the following formula:

$$
\begin{aligned}
& d(i, j) \\
& \quad=\sqrt{\left((f(1, i)-g(1, j)) \times(f(1, i)-g(1, j))^{\prime}\right)},
\end{aligned}
$$

where $f$ is the feature vector of the target subblock image and $g$ is the feature vector of the template subblock image.

Since there are 4 feature regions in each face image, we can get $4 d$ in each set of matching results. This paper uses a simple weighted method to fuse these $4 d$. Finally, the minimum is taken for the matching results.

\section{Experimental Results and Analysis}

The proposed 3D face recognition (Anthropometric Curvelet Fusion Face Recognition, ACFFR) is demonstrated in Texas 3D database which includes 1149 depth images of 118 persons with different expression, light, sex, and age.

4.1. Selection of Testing Sets and Training Sets. According to the evaluation standard of international human face recognition system, FRV2002 [16, 17] and FRGC2006 [18, 19], the Texas $3 \mathrm{D}$ database is divided into testing sets and training sets shown in Table 3. We randomly select 15 persons' images from Texas 3D database as training set to optimize the classifier. And each person has 30 images which include neutral images and expression images. Testing sets include 699 images of 103 persons, independent of training set, and testing sets are divided into model sets and target subsets. Model sets have 103 persons and each one has 1 neutral image. Target subset includes 600 images of 95 persons included in those 103 persons with neutral images and expression images.

4.2. Experiment. The statistics of ROC (Receiver Operating Characteristics) and EER (equal error rates) in the experiment are, respectively, shown in Figure 8 and Table 4 . The statistics of CMC (Cumulative Match Characteristic) and $\mathrm{RR}$ (rank 1 recognition rates) in recognition experiment are, respectively, shown in Figure 9 and Table 5. In order to demonstrate the effectiveness of the proposed algorithm, we make the comparison with the present famous methods in $[9,11]$. 
TABLE 4: The equal error rate of different algorithms.

\begin{tabular}{lccc}
\hline Algorithm/expression & Neutral & Expression & All \\
\hline Anthroface3D [12] & 1.65 & 2.81 & 1.98 \\
Curveletface3D [10] & 1.63 & 2.23 & 2.12 \\
ACFFR & 1.64 & 2.13 & 1.76 \\
\hline
\end{tabular}

TABLE 5: The recognition rate of different algorithms.

\begin{tabular}{lccc}
\hline Algorithm/expression & Neutral & Expression & All \\
\hline Anthroface3D [12] & 97.3 & 95.6 & 96.8 \\
Curveletface3D [10] & 99.4 & 94.1 & 97.1 \\
ACFFR & 97.5 & 96.0 & 97.0 \\
\hline
\end{tabular}

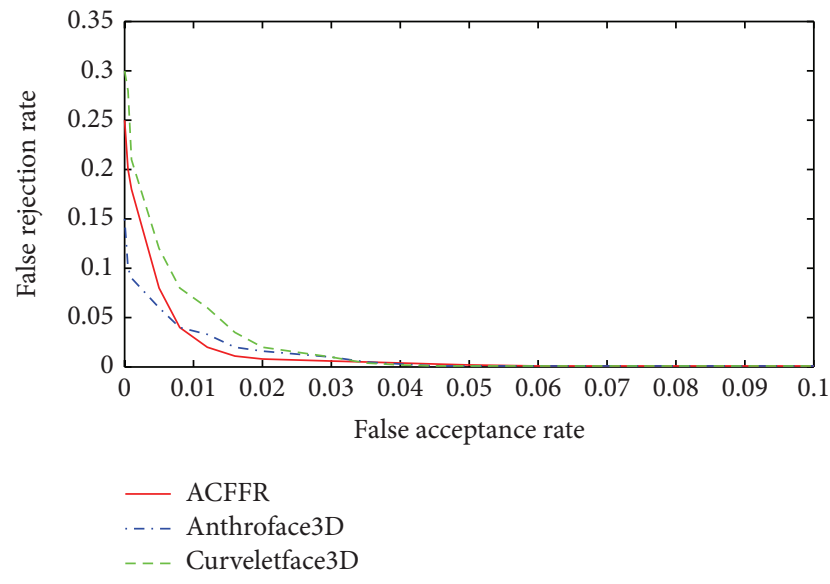

FIGURE 8: ROC curve.

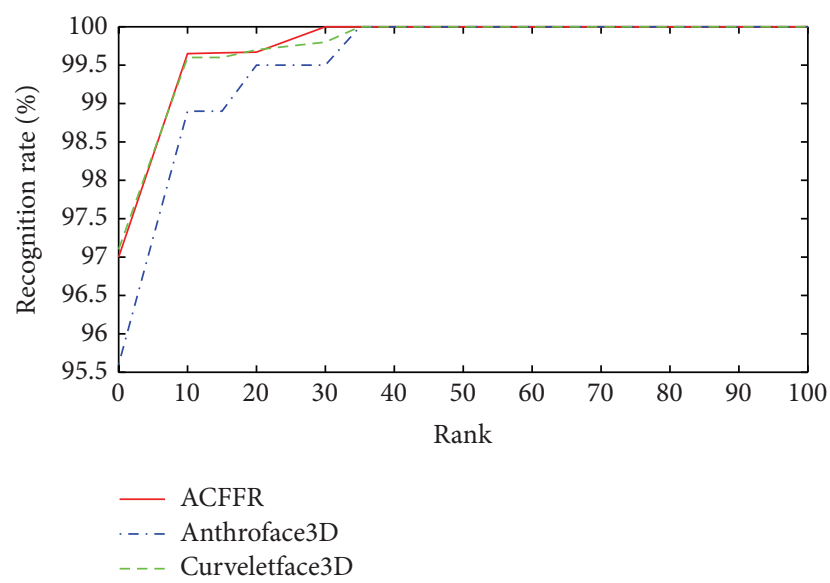

Figure 9: CMC curve.

From Figures 8 and 9, the proposed algorithm, ACFFR, is better than Anthroface3D and Curveletface3D. Statistics in Tables 4 and 5 have shown that EER is $1.75 \%$ and RR is $97.0 \%$ in our algorithm. Compared with Anthroface3D, the EER of ACFFR is lower and the best recognition rate (RR) is higher. Compared with Curveletface3D, the EER of ACFFR is slightly higher in neutral images while the EER of ACFFR is lower in expression images. Thus, the effectiveness of ACFFR is better than Anthroface3D and Curveletface3D on the occlusion and the expression changes.

The experiment result shows that the proposed Anthropometric and Curvelet features fusion-based algorithm for $3 \mathrm{D}$ face recognition (ACFFR) has fused 3D Curvelet features on the feature region based on Anthropometric features. Thus, it has achieved high performance.

\section{Conclusion}

This paper proposes an Anthropometric and Curvelet features fusion-based algorithm for 3D face recognition (Anthropometric Curvelet Fusion Face Recognition, ACFFR). The experiments have been done on the Texas 3D FR database. Experimental results show the superiority of ACFFR over Anthroface3D and Curveletface3D with EER rates of $2.12 \%$ and $\mathrm{RR}$ rates of $96.1 \%$ against EER rates of $2.81 \%$ and $2.23 \%$ and $R R$ rates of $95.6 \%$ and $94.1 \%$ in expressive images. Meanwhile, EER rate of ACFFR is $1.75 \%$ and $\mathrm{RR}$ rate of ACFFR is $97.0 \%$ for all face images including neutral images and expressive images. Thus, the ACFFR algorithm has good robustness to the occlusion and the changes of light and expression.

\section{Conflict of Interests}

The authors declare that there is no conflict of interests regarding the publication of this paper.

\section{Acknowledgments}

The authors are grateful to the anonymous reviewers who made constructive comments. This work is supported by the National Natural Science Foundation of China (no. 61203302 and no. 61403277) and the Tianjin Research Program of Application Foundation and Advanced Technology (14JCYBJC18900).

\section{References}

[1] S. Berretti, A. Del Bimbo, and P. Pala, "3D face recognition using isogeodesic stripes," IEEE Transactions on Pattern Analysis and Machine Intelligence, vol. 32, no. 12, pp. 2162-2177, 2010.

[2] K. Choudhary and N. Goel, "A review on face recognition techniques," in Proceedings of the International Conference on Communication and Electronics System Design (ICCESD '13), vol. 8760 of Proceedings of SPIE, Jaipur, India, January 2013.

[3] L. Xiaoli and D. Feipeng, "A rapid method for 3D face recognition based on rejection algorithm," Acta Automatica Sinica, vol. 36, no. 1, pp. 153-158, 2010 (Chinese).

[4] J. $\mathrm{Xu}$ and F.-P. Da, "3D face recognition based on local descriptor," Pattern Recognition and Artificial Intelligence, vol. 25, no. 1, pp. 45-53, 2012 (Chinese).

[5] V. P. Kshirsagar, M. R. Baviskar, and M. E. Gaikwad, "Face recognition using eigenfaces," in Proceedings of the 3rd International Conference on Computer Research and Development (ICCRD '11), vol. 2, pp. 302-306, IEEE, Shanghai, China, March 2011. 
[6] P. N. Belhumeur, J. P. Hespanha, and D. J. Kriegman, "Eigenfaces vs. fisherfaces: recognition using class specific linear projection," IEEE Transactions on Pattern Analysis and Machine Intelligence, vol. 19, no. 7, pp. 711-720, 1997.

[7] H. Mohammadzade and D. Hatzinakos, "Iterative closest normal point for 3D face recognition," IEEE Transactions on Pattern Analysis and Machine Intelligence, vol. 35, no. 2, pp. 381-397, 2013.

[8] C. Zhong, Z. Sun, and T. Tan, "Robust 3D face recognition using learned visual codebook," in Proceedings of the IEEE Computer Society Conference on Computer Vision and Pattern Recognition (CVPR '07), Minneapolis, Minn, USA, June 2007.

[9] S. Elaiwat, M. Bennamoun, F. Boussaid, and A. El-Sallam, "A curvelet-based approach for textured 3D face recognition," Pattern Recognition, vol. 48, no. 4, pp. 1235-1246, 2015.

[10] S. Elaiwat, M. Bennamoun, F. Boussaid, and A. El-Sallam, "3D face recognition using curvelet local features," IEEE Signal Processing Letters, vol. 21, no. 2, pp. 172-175, 2014.

[11] S. Gupta, M. K. Markey, and A. C. Bovik, "Anthropometric 3D face recognition," International Journal of Computer Vision, vol. 90, no. 3, pp. 331-349, 2010.

[12] S. Gupta, K. R. Castleman, M. K. Markey, and A. C. Bovik, "Texas 3D face recognition database," in Proceedings of the IEEE Southwest Symposium on Image Analysis and Interpretation (SSIAI '10), pp. 97-100, Austin, Tex, USA, May 2010.

[13] L. G. Farkas, Anthropometric Facial Proportions in Medicine, Thomas Books, 1987.

[14] L. G. Farkas, Ed., Anthropometry of the Head and Face, Raven Press, New York, NY, USA, 1994.

[15] D. L. Donoho and M. R. Duncan, "Digital curvelet transform: strategy, implementation, and experiments," in Wavelet Applications VII, vol. 4056 of Proceedings of SPIE, pp. 12-29, International Society for Optics and Photonics, Orlando, Fla, USA, April 2000.

[16] P. J. Phillips, P. Grother, R. J. Micheals, D. M. Blackburn, E. Tabassi, and J. M. Bone, "FRVT2002: Evaluation Report[EB/OL]".

[17] 2002, http://www.frvt.org/FRVT2002/documents.htm.

[18] P. J. Phillips, W. T. Scruggs, A. J. O’Toole et al., "FRVT 2006 and ICE 2006 large-scale results[EB/OL]," NISTIR 7408, National Institute of Standards and Technology, Gaithersburg, Md, USA, 2007.

[19] 2007, http://www.frvt.org/FRVT2006/default.aspx. 


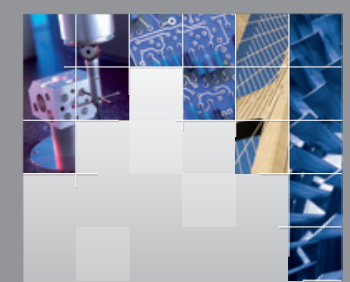

\section{Enfincering}
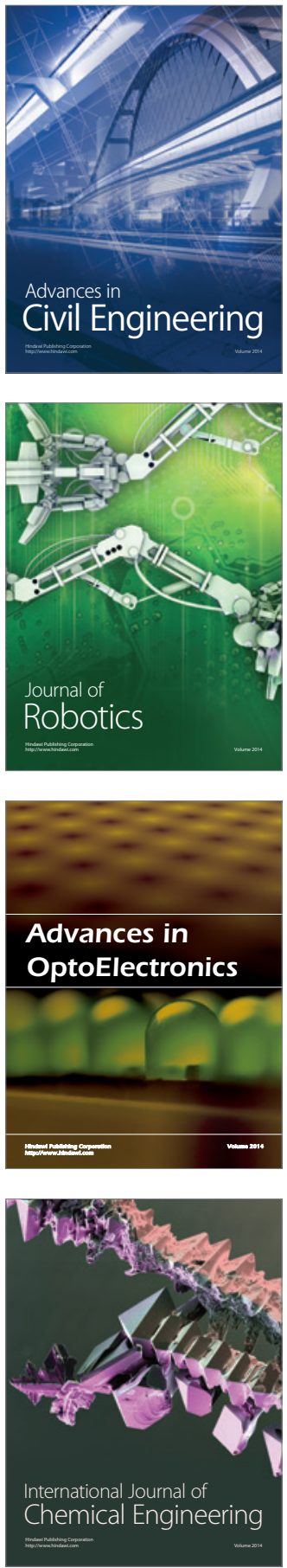

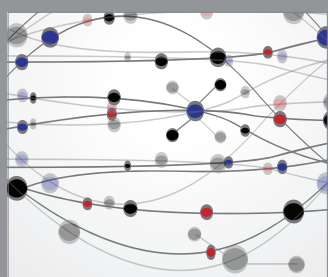

The Scientific World Journal

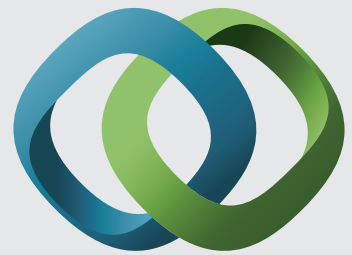

\section{Hindawi}

Submit your manuscripts at

http://www.hindawi.com
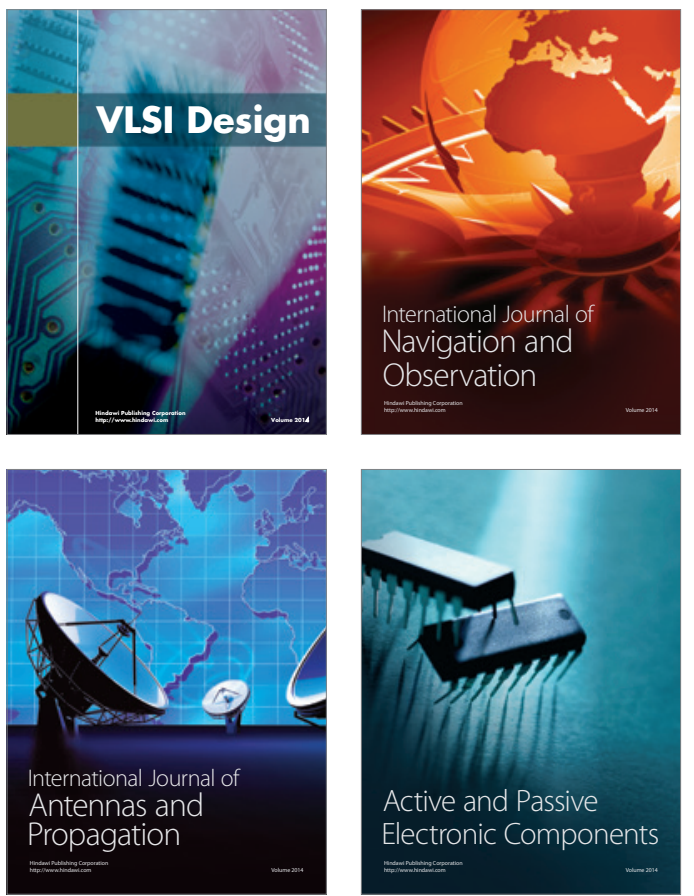
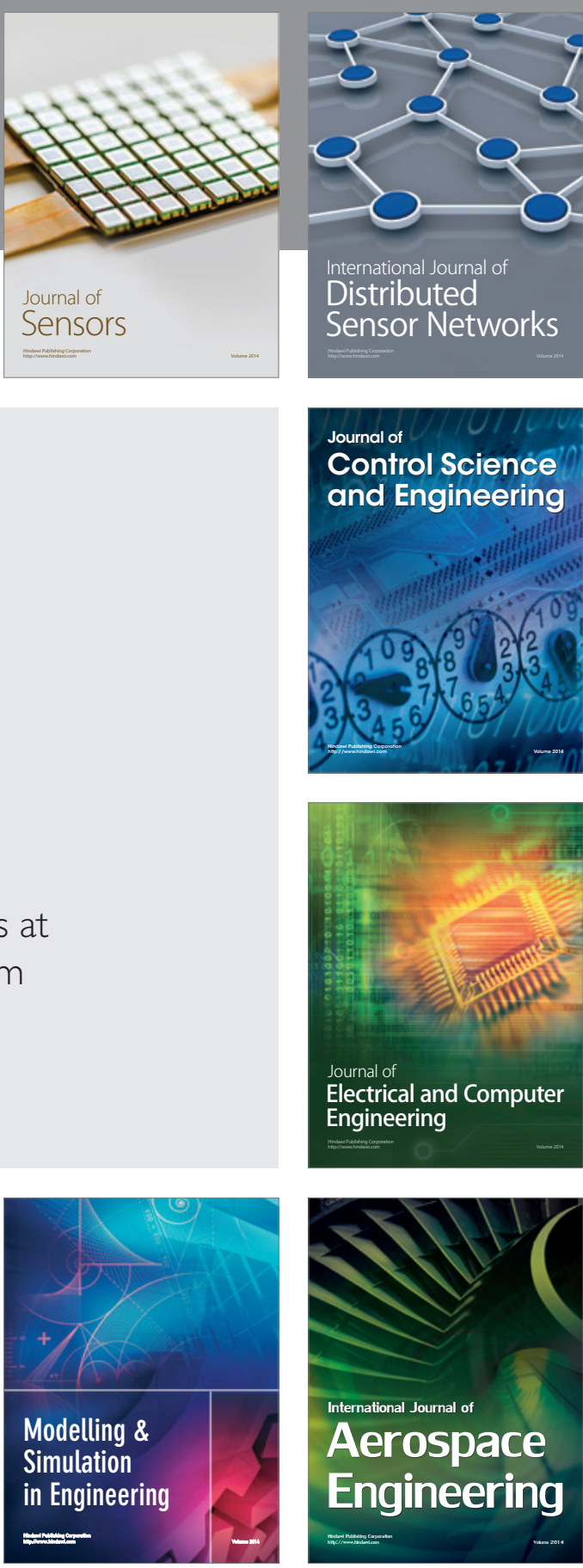

International Journal of

Distributed

Sensor Networks

Journal of

Control Science

and Engineering
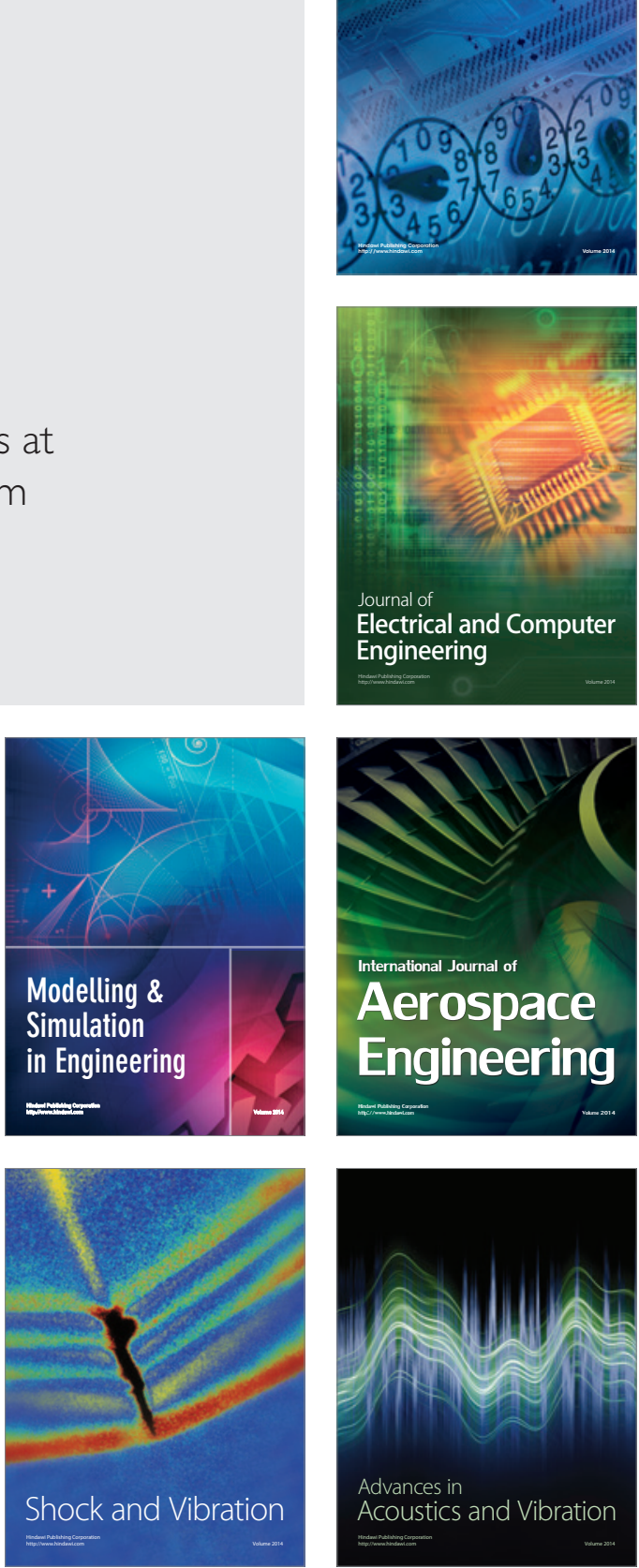